\title{
KINETIC MODELS OF CONSERVATIVE ECONOMIES WITH WEALTH REDISTRIBUTION*
}

\author{
MARZIA BISI ${ }^{\dagger}$, GIAMPIERO SPIGA $^{\ddagger}$, AND GIUSEPPE TOSCANI ${ }^{\S}$
}

\begin{abstract}
We introduce and discuss kinetic models for wealth distribution in a simple market economy, which are able to reproduce the salient features of the wealth distribution by including taxes to each trading process and redistributing the collected money among the population according to a given criterion. Our analysis gives a theoretical basis to some recent research that analyzed discrete simplified models for the exploitation of finite resources by interacting agents, where each agent receives a random fraction of the available resources. It is shown that in general the redistribution is able to modify the Pareto index, and that this modification can be quantified in terms of the redistribution operator.
\end{abstract}

Key words. Wealth and income distributions, kinetic models, Boltzmann equation, taxation and redistribution.

AMS subject classifications. 91B60, 82C40, 35B40.

\section{Introduction}

In recent years, there have been several attempts to model simple economies in which a wealth exchange process produces a distribution of wealth similar to that observed in real economies. One class that might be considered to constitute a mesoscopic approach is based on generalized Lotka-Volterra models [20, 26]. A second more popular approach relies on methods borrowed from statistical mechanics for particle systems $[18,12,6,5,17,25,9,11]$. Most of these works are particularly interested in microscopic models of markets where the economic activity is considered as a scattering process and the evolution of wealth obeys a kinetic equation of Boltzmann type. The features typically incorporated in kinetic trade models are saving effects and randomness. Saving means that each agent is guaranteed to retain at least a certain minimal fraction of his initial wealth at the end of the trade. This concept has been introduced in [6], where a fixed saving rate for all agents has been proposed, and generalized in [7] by introducing a individual saving rate. Randomness means that the amount of money changing hands is non-deterministic. Among others, this idea has been developed in [11] to include the effects of a risky market. Numerous numerical simulations for models of the prescribed type have been carried out with different mechanisms for saving and varying degree of randomness (see the recent book [8] for an overview of the recent results).

In most of the models introduced so far, the trading mechanism leaves the total mean wealth unchanged. Then, a substantial difference on the final behavior of the model (presence or not of tailed steady states) can be observed depending on the fact that binary trades are pointwise conservative, or conservative in the mean $[21,13]$. The asymptotic distribution of wealth, however, depends completely on the microscopic structure of binary trades. Other kinetic models have been recently proposed,

\footnotetext{
${ }^{*}$ Received: April 7, 2009; accepted (in revised version): September 8, 2009. Communicated by Lorenzo Pareschi.

†Dipartimento di Matematica, Università degli studi di Parma, 43100 Parma, Italy (marzia.bisi@unipr.it).

${ }^{\ddagger}$ Dipartimento di Matematica, Università degli studi di Parma, 43100 Parma, Italy (giampiero.spiga@unipr.it).

$\S$ Dipartimento di Matematica, Università degli studi di Pavia, 27100 Pavia, Italy (giuseppe.toscani@unipv.it).
} 
which, while maintaining the kinetic description, introduce more sophisticated rules for trading. For example, a description of the behavior of a stock price has been developed by Cordier, Pareschi and Piatecki in [10]. Further, there have been efforts to include non-microscopic effects, like global taxation (and subsequent redistribution), in recent works of Guala [16], Pianegonda, Iglesias, Abramson, and Vega [24], and Garibaldi, Scalas, and Viarengo [15].

The model introduced in [16] studies how inelastic binary collisions may be assumed as the application of taxes, and how its redistribution can reproduce the salient features of empirical distributions of wealth. The model is reminiscent of the inelastic kinetic model introduced by Slanina [25], and takes into account a simple granular closed-system model in which the collisions are inelastic and the loss of energy is eventually redistributed among the particles of the system according to a certain criterion.

In [24], the market is modeled as a one-dimensional lattice, every site of which represents an agent. Agents with closer ties to each other will be neighbors on the lattice. Starting from an initial configuration where the wealth is distributed randomly among agents, all agents strive to improve their situation. In particular the poorest agent is the one feeling the strongest pressure to move up the ladder. At each time step, the poorest agent, i.e., the one with the minimum wealth, will take some action to improve its economic state. Since the outcome of any such measure is uncertain, this outcome is modeled as a random change in the wealth parameter of this agent. Moreover, whatever wealth is gained (lost) by the poorest agent will be at the expense of its neighbors and it is assumed to be equally divided among its two nearest neighbors. After a relatively long transient the system arrives at a stationary wealth distribution.

Finally, in [15] a simple stochastic exchange game mimicking taxation and redistribution has been studied. Starting from an initial configuration where the wealth is distributed randomly among agents, taxation is modeled by randomly extracting some agents. Then, their wealths are redistributed to agents following Polya's scheme. The individual wealth equilibrium distribution is subsequently shown to converge, in the continuum limit, to a Gamma distribution, whose form factor is just the initial redistribution weight.

The object of this paper is to show how most of these discrete results can be modeled at a continuous level owing to a kinetic equation of Boltzmann type, similar to the ones introduced in [21]. The novelty is to introduce a simple taxation mechanism at the level of the single trade to generate a portion of the mean wealth of the society, that will be totally redistributed to agents to maintain the total wealth constant. The mechanism of redistribution is assumed sufficiently flexible to be able to redistribute to agents a constant amount of wealth independently of the wealth itself, or to redistribute proportionally (or inversely proportionally) to their wealth.

The paper is organized as follows. In the next section we introduce the model which is described by a Boltzmann equation with a drift, and its main features are discussed in some detail. Mathematical aspects related to the well posedness of the problem are analyzed preliminarily in section 3. Section 4 deals with the connections that may be established between our model problem with taxation and redistribution and the well known non-conservative model by Slanina [25], in which an exact analytical solution with Pareto tails is available for special values of parameters. The main results of our model for general values of transaction and redistribution parameters are presented in sections 5 and 6 , with particular emphasis on derivation and clas- 
sification of steady states in suitable continuous trade (hydrodynamic) limits. The considered scenarios include stochastic models with risks and deterministic models without risks, and in all cases explicit dependence of Pareto index on coefficients is achieved. Numerical results on the solution of the kinetic equation are scheduled as future work.

\section{Kinetic models with redistribution}

The goal of a kinetic model of a simple market economy is to describe the evolution of the distribution of wealth by means of microscopic interactions among agents or individuals which exchange money. In this picture each trade is interpreted as an interaction where a fraction of the money changes hands. One generally assumes that this wealth after the interaction is non negative, which corresponds to impose that no debts are allowed. In consequence of the trade rules, it is expected to obtain for large time a stationary wealth distribution $f_{\infty}(v)$ (denoting the density of agents with wealth $v>0$ ) with Pareto tails,

$$
F_{\infty}(w)=\int_{w}^{\infty} f_{\infty}(v) d v \propto w^{-\alpha} .
$$

The exponent $\alpha$ is referred to as Pareto index, named after the economist Vilfredo Pareto [23], who proposed formula (2.1) more than a hundred years ago. According to recent empirical data, the wealth distribution among the population in a western country follows in fact a Pareto law, with an index $\alpha$ ranging between 1.5 and 2.5.

The study of the time-evolution of the wealth distribution among individuals in a simple economy, together with a reasonable explanation of the formation of tails in this distribution has been recently achieved by means of kinetic collision-like models in [21] (see also [13]). The time evolution of the wealth distribution $f(v, t)$ is based on the assumption of collision-like trade events. In a suitable scaling, the effect of collisions is quantitatively described by a Boltzmann-like equation

$$
\frac{\partial f}{\partial t}=Q(f, f) .
$$

Here $f=f(v, t)$ is the distribution of wealth $v \in \mathbb{R}_{+}$at time $t \geq 0$, and $Q$ is a bilinear operator which describes the change of $f$ due to trades among agents. The binary trade is determined by the linear exchange rules

$$
v^{*}=p_{1} v+q_{1} w, \quad w^{*}=p_{2} v+q_{2} w .
$$

Here $(v, w)$ denote the (positive) money of two arbitrary individuals before the trade, and $\left(v^{*}, w^{*}\right)$ the money after the trade. The transaction coefficients $p_{i}, q_{i}, i=1,2$ can be either given constant or random quantities, with the obvious constraint to be nonnegative. In the present paper the random distribution (or collision kernel, in the kinetic language) is assumed to be independent of the wealth variables $v$ and $w$ and independent of time. Moreover, we restrict ourselves to conservative models, characterized by the further property

$$
\left\langle p_{1}+p_{2}\right\rangle=1, \quad\left\langle q_{1}+q_{2}\right\rangle=1,
$$

where $\langle\cdot\rangle$ denotes the expectation value. In consequence of $(2.4)$, the total wealth involved in the trade (2.3) is conserved in the mean

$$
\left\langle v^{*}+w^{*}\right\rangle=v+w .
$$


A number of different trade models fit into this class. The first by Chakraborti and colleagues $[6,5]$ conserves money during the exchange and allows savings that can be a fixed and equal percentage of the initial money held by each agent. Allowing the saving percentage to take on a random character [7] introduces then a power law character to the distribution for high incomes that can be shown to allow existence of power moments only up to exactly order one. The presence of random terms in the trade, introduced by Cordier, Pareschi and one of the authors [11] which destroy the pointwise conservation of wealth, was subsequently shown to be responsible of a robust convergence to a steady distribution with tails [21].

The homogeneous Boltzmann equation (2.2) can easily be written in weak form. It corresponds to saying that the solution to (2.2) satisfies, for all smooth functions $\phi(v)$,

$$
\frac{d}{d t} \int_{\mathbb{R}_{+}} f(v) \phi(v) d v=\left\langle\int_{\mathbb{R}_{+}^{2}}\left(\phi\left(v^{*}\right)-\phi(v)\right) f(v) f(w) d v d w\right\rangle .
$$

Note that (2.6) implies that $f(v, t)$ remains a probability density if it is so initially

$$
\int_{\mathbb{R}_{+}} f(v, t) d v=\int_{\mathbb{R}_{+}} f_{0}(v) d v=1
$$

Moreover, owing to (2.4), the total mean wealth is also preserved in time

$$
m(t)=\int_{\mathbb{R}_{+}} v f(v, t) d v=\int_{\mathbb{R}_{+}} v f_{0}(v) d v=m(0) .
$$

Since our goal is to understand the role of redistribution, in what follows we assume that a percentage of the total wealth involved in the trade is not returned to agents. We can think at this small fraction that does not return as a taxation operated on the trade. In this case, for a given positive constant $0<\epsilon<1$, the post-interaction wealths satisfy

$$
\left\langle v^{*}+w^{*}\right\rangle=(1-\epsilon)(v+w) .
$$

This condition can be reached in a variety of ways. Let us assume that the transaction coefficients $p_{i}, q_{i}, i=1,2$ are bounded from below

$$
\min _{i=1,2}\left\{p_{i}, q_{i}\right\}>\delta
$$

for a given small constant $\delta>0$. Inequality (2.10) implies that a trade can take place only if both agents participate by putting part of their money into the game. Then, for any positive constant $\epsilon \leq \delta$, the trade

$$
v_{\epsilon}^{*}=\left(p_{1}-\epsilon\right) v+q_{1} w, \quad w_{\epsilon}^{*}=p_{2} v+\left(q_{2}-\epsilon\right) w
$$

is such that both $v^{*}$ and $w^{*}$ are nonnegative, but conservation of wealth is lost

$$
\left\langle v_{\epsilon}^{*}+w_{\epsilon}^{*}\right\rangle=(1-\epsilon)(v+w) .
$$

We remark that condition (2.10) is enough to prove Theorem 3.5 in [21], which implies the existence of a unique regular steady state to the Boltzmann equation (2.2). This is clearly an oversimplified way to introduce taxation into the binary trade rule. The 
main economic reason behind this choice is to prevent from the beginning, by fixing an upper bound to the taxation parameter, the possibility that the percentage of taxation will be greater than the minimal quantity of wealth any agent can put into the trade. Of course, different ways of taxation produce the same effect. For example

$$
\tilde{v}_{\epsilon}^{*}=p_{1} v+q_{1} w-\frac{\epsilon}{2}(v+w), \quad \tilde{w}_{\epsilon}^{*}=p_{2} v+q_{2} w-\frac{\epsilon}{2}(v+w),
$$

and

$$
\check{v}_{\epsilon}^{*}=(1-\epsilon)\left(p_{1} v+q_{1} w\right), \quad \check{w}_{\epsilon}^{*}=(1-\epsilon)\left(p_{2} v+q_{2} w\right),
$$

also produce (2.9). In the rest of the paper we will deal with trades of type (2.11). Our analysis, however, can easily be extended without any additional difficulty to the other types of trades.

Let $Q_{\epsilon}(f, f)$ define the collision operator governing the non-conservative process which corresponds to the trade (2.11). In weak form,

$$
\frac{d}{d t} \int_{\mathbb{R}_{+}} f(v) \phi(v) d v=\left\langle\int_{\mathbb{R}_{+}^{2}}\left(\frac{\phi\left(v_{\epsilon}^{*}\right)+\phi\left(w_{\epsilon}^{*}\right)-\phi(v)-\phi(w)}{2}\right) f(v) f(w) d v d w\right\rangle .
$$

Note that, due to (2.12), the total mean wealth, on account of (2.4), is exponentially decaying in time

$$
m(t)=\int_{\mathbb{R}_{+}} v f(v, t) d v=m(0) \exp \{-\epsilon t\} .
$$

The percentage of mean wealth that comes out by taxation, can be restituted to the agents in such a way that the total wealth is left unchanged. This can be done by resorting to a redistribution operator, which we can assume to be of the form

$$
R_{\chi}^{\epsilon}(f)(v, t)=\epsilon \frac{\partial}{\partial v}[(\chi v-(\chi+1) m(t)) f(v, t)] .
$$

It is meant here that $m(t)$ denotes the first power moment of $f$, which, in general, makes the operator $R_{\chi}^{\epsilon}$ nonlinear. The weight factor multiplying the distribution function inside the square brackets in (2.17) has been taken to be linear in $v$ for simplicity, also in order to involve in the mechanism only the most meaningful moments, namely those of order zero and one. Such a weight function contains only one disposable real parameter $\chi$, a constant which characterizes the type of redistribution, and that determines the slope of the straight line as well as the value of $v$, no matter if physical or non-physical, at which the weight itself vanishes. The other parameter has been determined by the constraint that the redistribution operator preserves the number of agents and actually redistributes the total amount of money that is being collected by taxation. In fact, note that we have, whatever the constant $\chi$,

$$
\int_{\mathbb{R}_{+}} v R_{\chi}^{\epsilon}(f)(v, t) d v=\epsilon m(t),
$$

and, provided $f(v, t)$ satisfies in addition the "boundary" condition $f(0, t)=0$, also

$$
\int_{\mathbb{R}_{+}} R_{\chi}^{\epsilon}(f)(v, t) d v=0 .
$$


The additional boundary condition would not be needed in the special case $\chi=-1$, which reproduces the standard anti-drift operator considered by Slanina [25]. It is well known that, in this situation, the steady wealth distribution has thick tails.

A further insight into the effect of the operator (2.17) comes out from the analytic expression of the solution to the equation

$$
\frac{\partial}{\partial t} f(v, t)=R_{\chi}^{\epsilon}(f)(v, t),
$$

with initial density $f_{0}(v)$ and corresponding initial average wealth $m(0)$. First, it is immediately realized that the process actually creates wealth, with $m(t)=m(0) \exp (\epsilon t)$. That renders the kinetic equation (2.20) linear, and it can be verified by direct inspection that the solution reads

$$
f(v, t)=e^{\epsilon \chi t} f_{0}\left[e^{\epsilon \chi t} v-m(0)\left(e^{\epsilon(\chi+1) t}-1\right)\right], \quad t>0 .
$$

Let us remark that, if $f_{0}(v)=0$ for $v<0$, the solution at time $t>0$ vanishes for

$$
v<m(0) \frac{e^{\epsilon(\chi+1) t}-1}{e^{\epsilon} \chi^{t}},
$$

which implies that no agents with wealth below the right-hand side of (2.22) are present at time $t$. For $\chi>-1$ the threshold increases in time and diverges as $O\left(e^{\epsilon t}\right)$ for $t \rightarrow+\infty$.

It is not difficult to check that the actual redistribution function among the population of agents (namely the normalized fraction $\psi(v)$ of the total collected money that is supplied to each agent of wealth $v$ ) can be deduced from (2.17) and reads as

$$
\psi(v, t) \frac{\partial f}{\partial v}=-\left[\frac{\chi}{m(t)} v-(\chi+1)\right] \frac{\partial f}{\partial v}-\frac{\chi}{m(t)} f(v, t) .
$$

Clearly $\psi$ depends on $f$ in a complicated way, however its qualitative analysis versus $v$ is sufficient for an estimate of the actual redistribution to agents with different wealths when the characteristic parameter $\chi$ of the operator $R_{\chi}^{\epsilon}$ is varied. The case in which the total yield from taxation is equally distributed among all agents corresponds to the option $\psi(v)=1$, and is achieved in (2.17) by the special option $\chi=0$. In all other cases the redistribution is selective, and may correspond to some partition strategy. It is not guaranteed that $\psi$ should be positive for any $v$, namely the redistribution might take away further money (beyond taxation) from a part of the population, and reinforce another part. On the other hand, in this model the processes described by the two distinct operators introduced so far proceed simultaneously, and gain or loss is determined by the overall balance only. It is easy to see that for the most reasonable trends for the wealth profiles $f$, the redistribution function $\psi$ is negative or positive in a right neighborhood of $v=0$ according to whether $\chi<-1$ or $\chi>-1$, respectively. Analogously, when $v$ is large enough, $\psi$ turns out to be positive or negative for $\chi<0$ or $\chi>0$, respectively. In a sense, for positive values of the parameter $\chi$, money is redistributed to agents with little wealth, whereas agents of large wealth are taxed once more. When $\chi<-1$, we would have the opposite situation in which the poorest part of the population supplies additional resources to the richest part, and for this reason we will exclude this range of parameter values from the analysis below. Finally, in the intermediate case $-1<\chi<0$, both the richest and poorest agents are favored by the taxation/redistribution mechanism, at the expenses of the "middle class". 
In the next sections we will study in detail the time-evolution to the solution of the kinetic equation with taxation/redistribution

$$
\frac{\partial f(v, t)}{\partial t}=Q_{\epsilon}(f, f)(v, t)+R_{\chi}^{\epsilon}(f)(v, t),
$$

where the bilinear operator $Q_{\epsilon}$ accounts for taxation in trades, while the differential operator $R_{\chi}^{\epsilon}$ accounts for redistribution and (possible) additional taxation. It is clear, and immediately checked by integration, that the process (2.24) governed by the full operator $Q_{\epsilon}+R_{\chi}^{\epsilon}$ not only preserves the number of agents, but also is globally conservative in the mean, namely $\frac{d}{d t} \int_{\mathbb{R}_{+}} v f(v, t) d v=0$, so that now the average wealth remains constant, $m(t)=m(0)=m$.

\section{Preliminary results}

Without loss of generality, we can fix the initial density to satisfy

$$
\int_{\mathbb{R}_{+}} f_{0}(v) d v=1, \quad \int_{\mathbb{R}_{+}} v f_{0}(v) d v=m .
$$

As obtained in the previous section, conditions (3.1) are propagated in time, so that, if $f(v, t)$ denotes the solution to $(2.24)$, at least formally for all $t>0$

$$
\int_{\mathbb{R}_{+}} f(v, t) d v=1, \quad \int_{\mathbb{R}_{+}} v f(v, t) d v=m
$$

The proof of the first equality in (3.2) (mass conservation) requires that (2.19) holds, and this requires that $f(v, t)=0$ in $v=0$. Even if this appears as a reasonable boundary condition for our problem (in that it implies that if no agents with negative wealth are allowed to trade, the resulting density does not develop a jump at the boundary $v=0$ ), it is not immediate to conclude that this condition, assumed at time $t=0$, propagates at any subsequent time $t>0$. Using standard methods of kinetic theory, however, one can see that this property holds true for the kinetic equation (2.24), provided the initial density $f_{0}(v)$ is sufficiently regular.

As it is usually done for this type of kinetic models, most of the analytical properties of the solution are obtained by means of Fourier analysis. From the kinetic theory of rarefied gases it is well-known that Boltzmann-like equations are often conveniently studied in Fourier space, see e.g. [3]. This is particularly true for the model under consideration, since its collision kernel (the probability measure) is independent of $v$. In fact, the weak formulation (2.15) for equation (2.24) is equivalent to the Fourier transformed equation

$$
\frac{\partial \widehat{f}(\xi, t)}{\partial t}=\widehat{Q_{\epsilon}}(\widehat{f}, \widehat{f})(\xi, t)+\widehat{R_{\chi}^{\epsilon}}(\widehat{f})(\xi, t)
$$

where $\widehat{f}(\xi, t)$ is the Fourier transform of $f(v, t)$,

$$
\widehat{f}(\xi, t)=\int_{\mathbb{R}_{+}} e^{-i \xi v} f(v, t) d v .
$$

According to the collision rule (2.11), the transformed kernel reads

$$
\widehat{Q_{\epsilon}}(\widehat{f}, \widehat{f})(\xi)=\frac{1}{2}\left\langle\widehat{f}\left(\left(p_{1}-\epsilon\right) \xi\right) \widehat{f}\left(q_{1} \xi\right)+\widehat{f}\left(p_{2} \xi\right) \widehat{f}\left(\left(q_{2}-\epsilon\right) \xi\right)\right\rangle-\widehat{f}(\xi) \widehat{f}(0),
$$


while

$$
\widehat{R_{\chi}^{\epsilon}}(\widehat{f})(\xi, t)=-\epsilon \chi \xi \frac{\partial}{\partial \xi} \widehat{f}(\xi, t)-i \epsilon(\chi+1) m(t) \xi \widehat{f}(\xi, t) .
$$

The initial conditions (3.1) turn into

$$
\widehat{f_{0}}(0)=1 \text { and }{\widehat{f_{0}}}^{\prime}(0)=-i m .
$$

To prove existence and uniqueness of solutions to equation (3.3) is rather classical [21]. Indeed, our model differs from the pure kinetic equation treated in [21] for the presence of the redistribution operator (3.5) which corresponds, due to (2.21), to the action of both shift and drift operators, which affect neither existence nor uniqueness of solutions. In addition, under condition (2.10), regularity can also be shown to be propagated in time. To this aim, let us consider the Sobolev spaces of high degree

$$
\|g\|_{\dot{H}^{\eta}(\mathbb{R})}^{2}=\int_{\mathbb{R}}|\xi|^{2 \eta}|\hat{g}(\xi)|^{2} d \xi
$$

with $\eta>0$. Indeed, we obtain

$$
\begin{aligned}
\frac{d}{d t}\|f(t)\|_{\dot{H}^{\eta}}^{2} & =\frac{d}{d t} \int_{\mathbb{R}}|\xi|^{2 \eta} \hat{f}(\xi, t) \overline{\hat{f}(\xi, t)} d \xi \\
& =2 \int_{\mathbb{R}}|\xi|^{2 \eta} \operatorname{Re}\left(\overline{\hat{f}(\xi, t)} \partial_{t} \hat{f}(\xi, t)\right) d \xi \\
& =2 \int_{\mathbb{R}}|\xi|^{2 \eta} \operatorname{Re}\left(\overline{\hat{f}(\xi, t)}\left(\widehat{Q_{\epsilon}}(\widehat{f}, \widehat{f})(\xi, t)+\widehat{R_{\chi}^{\epsilon}}(\widehat{f})(\xi, t)\right)\right) d \xi .
\end{aligned}
$$

Consider now that, by mass conservation, $|\widehat{f}(\xi, t)| \leq 1$. Consequently

$$
\begin{aligned}
& \int_{\mathbb{R}}|\xi|^{2 \eta} \operatorname{Re}\left(\overline{\hat{f}(\xi, t)}\left\langle\hat{f}\left(\left(p_{1}-\epsilon\right) \xi, t\right) \hat{f}\left(q_{1} \xi, t\right)\right\rangle\right) d \xi \\
\leq & \int_{\mathbb{R}}|\xi|^{2 \eta}|\hat{f}(\xi, t)|\left\langle\left|\hat{f}\left(\left(p_{1}-\epsilon\right) \xi, t\right)\right|\left|\hat{f}\left(q_{1} \xi, t\right)\right|\right\rangle d \xi \\
\leq & \int_{\mathbb{R}}|\xi|^{2 \eta}|\hat{f}(\xi, t)|\left\langle\left|\hat{f}\left(q_{1} \xi, t\right)\right|\right\rangle d \xi=\left\langle\int_{\mathbb{R}}|\xi|^{2 \eta}|\hat{f}(\xi, t)|\left|\hat{f}\left(q_{1} \xi, t\right)\right| d \xi\right\rangle .
\end{aligned}
$$

Since the trade coefficient $q_{1}$ satisfies condition (2.10), $\left|q_{1}\right| \geq \delta$, and

$$
\begin{aligned}
& \int_{\mathbb{R}}|\xi|^{2 \eta}|\hat{f}(\xi, t)|\left|\hat{f}\left(q_{1} \xi, t\right)\right| d \xi \leq\left(\int_{\mathbb{R}}|\xi|^{2 \eta}|\hat{f}(\xi, t)|^{2} d \xi\right)^{1 / 2}\left(\int_{\mathbb{R}}|\xi|^{2 \eta}\left|\hat{f}\left(q_{1} \xi, t\right)\right|^{2} d \xi\right)^{1 / 2} \\
= & \left.\left.\left(\int_{\mathbb{R}}|\xi|^{2 \eta}|\hat{f}(\xi, t)|^{2} d \xi\right)^{1 / 2}\left|q_{1}\right|^{-\left(\eta+\frac{1}{2}\right)}\left|\int_{\mathbb{R}}\right| q_{1} \xi\right|^{2 \eta}\left|\hat{f}\left(q_{1} \xi, t\right)\right|^{2} d\left(q_{1} \xi\right)\right|^{1 / 2} \\
\leq & \delta^{-\left(\eta+\frac{1}{2}\right)} \int_{\mathbb{R}}|\xi|^{2 \eta}|\hat{f}(\xi, t)|^{2} d \xi
\end{aligned}
$$

An identical result holds for the second term of $\widehat{Q}_{\epsilon}$. Finally

$$
\int_{\mathbb{R}}|\xi|^{2 \eta} \operatorname{Re}\left(\overline{\hat{f}(\xi, t)} \widehat{Q_{\epsilon}}(\widehat{f}, \widehat{f})(\xi, t)\right) d \xi \leq\left(\delta^{-\left(\eta+\frac{1}{2}\right)}-1\right) \int_{\mathbb{R}}|\xi|^{2 \eta}|\hat{f}(\xi, t)|^{2} d \xi
$$


Moreover, integration by parts yields

$$
\begin{aligned}
& \int_{\mathbb{R}}|\xi|^{2 \eta} \operatorname{Re}\left(\overline{\hat{f}(\xi, t)} \widehat{R_{\chi}^{\epsilon}}(\widehat{f})(\xi, t)\right) d \xi=-\frac{\epsilon \chi}{2} \int_{\mathbb{R}} \xi|\xi|^{2 \eta} \frac{\partial}{\partial \xi}|\widehat{f}(\xi, t)|^{2} d \xi \\
= & \frac{\epsilon \chi}{2}(2 \eta+1) \int_{\mathbb{R}}|\xi|^{2 \eta}|\hat{f}(\xi, t)|^{2} d \xi .
\end{aligned}
$$

From (3.6) and (3.7) we obtain

$$
\frac{d}{d t}\|f(t)\|_{\dot{H}^{\eta}}^{2} \leq 2\left(\delta^{-\left(\eta+\frac{1}{2}\right)}-1+\frac{\epsilon \chi}{2}(2 \eta+1)\right)\|f(t)\|_{\dot{H}^{\eta}}^{2} .
$$

We proved:

Lemma 3.1. Let the initial density $f_{0}(v)$ belong to $\dot{H}^{\eta}$, for some $\eta>0$. Then, if the transaction coefficients satisfy (2.10), the unique solution to the kinetic equation (3.3) belongs to $\dot{H}^{\eta}$ at any subsequent time $t>0$, and the following bound holds

$$
\|f(t)\|_{\dot{H}^{\eta}} \leq \exp \left\{\left(\delta^{-\left(\eta+\frac{1}{2}\right)}-1+\frac{\epsilon \chi}{2}(2 \eta+1)\right) t\right\}\left\|f_{0}\right\|_{\dot{H}^{\eta}} .
$$

The result of Lemma 3.1 guarantees that the boundary condition $f(v=0, t)=0$ is preserved in time, provided it holds true at time $t=0$. Suppose indeed that the initial density for equation (3.3) is such that $f_{0}(v)=0$ if $v \leq 0$. Then, if $f_{0}(v) \in \dot{H}^{\eta}$, with $\eta>1 / 2$, the regularity, according to Lemma 3.1, is propagated in time. By the Sobolev imbedding [2], this regularity is enough to guarantee that the solution $f(v, t)$, for any $t>0$ is a bounded and continuous function. Since the trade mechanism (2.11) is such that the post-trade wealths are nonnegative, the wealth which is initially distributed on $\mathbb{R}_{+}$remains distributed on $\mathbb{R}_{+}$, so that $f(v, t)=0$ if $v<0$. On the other hand, since $f(v, t)$ is continuous in $v=0$, the condition $f(v=0, t)=0$ follows.

\section{A non-conservative model without risk}

Motivated by the analogy with a dissipative Maxwell gas, F. Slanina [25] introduced an increasing wealth model without distribution, of type (2.3), where

$$
p_{1}=1-q+\nu, \quad q_{1}=q ; \quad p_{2}=q, \quad q_{2}=1-q+\nu .
$$

In (4.1), the growth parameter $\nu$ is a fixed positive constant, which implies that the total wealth has increased after the trade,

$$
v^{*}+w^{*}=(1+\nu)(v+w) .
$$

Since the mean wealth corresponding to this model, labeled now as $M(t)$, is increasing exponentially in time, $M(t)=M(0) e^{\nu t}$, stationary solutions do not exist. However, the large-time behavior of the model can be described by self-similar solutions. The standard way to look for self-similarity is to scale the solution according to the rule

$$
g(v, t)=M(t) f(M(t) v, t)
$$

which implies that $\int_{\mathbb{R}_{+}} v g(v, t) d v=1$ for all $t \geq 0$. Moreover $g=g(v, t)$ satisfies the 
equation

$$
\begin{aligned}
& \frac{d}{d t} \int_{\mathbb{R}_{+}} \phi(v) g(v, t) d v-\nu \int_{\mathbb{R}_{+}} \phi(v) \frac{\partial}{\partial v}(v g) d v \\
= & \frac{1}{2} \int_{\mathbb{R}_{+}^{2}} g(v) g(w)\left(\phi\left(v^{*}\right)+\phi\left(w^{*}\right)-\phi(v)-\phi(w)\right) d v d w \\
= & \int_{\mathbb{R}_{+}^{2}} g(v) g(w)\left(\phi\left(v^{*}\right)-\phi(v)\right) d v d w,
\end{aligned}
$$

for any smooth function $\phi$. By definition, while the mean wealth of $f$ is increasing, the scaling (4.3) is such that the mean wealth of $g$ is kept constant. Hence, the drift operator in (4.4) is responsible for the stabilization of the mean wealth. Now suppose that $\nu=-\epsilon$. Then, the trade (4.1) coincides with the trade (2.11), the mean wealth is decreasing exponentially in time, and the scaling (4.3) implies again that the mean wealth of $g$ is kept constant. In this case, the drift operator in (4.4) coincides with the redistribution (2.17), corresponding to the choice $\chi=-1$.

Let us emphasize that, if $\nu=-\epsilon$, the problem of looking for a self-similar solution to the kinetic dissipative problem given by (4.1) can be rephrased in an equivalent way as the problem of looking for a stationary solution for a taxation-redistribution in which the redistribution $(\chi=-1)$ is such that the part of the population with intermediate-low income supplies additional resources to the richest part. As a consequence of this, the solution density tends to produce fat tails. This can be shown by resorting to the example of Slanina's paper [25], suitably modified to match our context. Setting as usual

$$
\tilde{g}(s, t)=\int_{\mathbb{R}_{+}} g(v, t) \exp (-s v) d v,
$$

the equation for $\tilde{g}$ is nothing but the weak form of the kinetic equation relevant to the test function $\phi(v)=\exp (-s v)$, with $s \in \mathbb{R}_{+}$

$$
\frac{\partial \tilde{g}}{\partial t}-\epsilon s \frac{\partial \tilde{g}}{\partial s}=\tilde{g}(q s) \tilde{g}((1-q-\epsilon) s)-\tilde{g}(s) .
$$

Steady solutions to equation (4.6) satisfy

$$
-\epsilon s \frac{\partial \tilde{g}}{\partial s}=\tilde{g}(q s) \tilde{g}((1-q-\epsilon) s)-\tilde{g}(s), \quad \tilde{g}(0)=1, \quad \tilde{g}^{\prime}(0)=-1 .
$$

Suppose

$$
q=\frac{1-\epsilon-\sqrt{1-2 \epsilon}}{2},
$$

with $\epsilon<\frac{1}{2}$, which implies $0<q<\frac{1}{4}$. Direct computations then show that

$$
\sqrt{2 q} \sqrt{2(1-\lambda-\epsilon)}=\epsilon, \quad \sqrt{q}+\sqrt{1-q-\epsilon}=1,
$$

and that the function

$$
\tilde{g}_{\infty}(s)=(1+\sqrt{2 s}) e^{-\sqrt{2 s}}
$$


solves (4.7). The function (4.9) is the Laplace transform of the generalized $\Gamma$ distribution $[4,11]$

$$
g_{\infty}(v)=\frac{1}{\sqrt{2 \pi}} \frac{\exp \left(-\frac{1}{2 v}\right)}{v^{5 / 2}} .
$$

This stationary distribution exhibits a Pareto power law tail for large $v$ 's, with Pareto index 3/2. Unfortunately, this analytical solution is valid only under the constraint (4.8), linking together transaction and taxation parameters, $q$ and $\epsilon$, and does not apply to the general case and to a general redistribution factor $\chi$.

\section{Conservative models with risk}

To our knowledge, the example presented in the previous section is the unique case in which an explicit solution to the kinetic model can be found. In all the other cases, it is quite difficult to obtain from the kinetic model quantitative results both on the shape of the resulting density, and on the effects of the redistribution operator. Leaving for the moment the problem of approximating the solution of the Boltzmann equation through numerical methods, here we resort to an asymptotic method first introduced in [11] that allows us to recover from the Boltzmann equation a related Fokker-Planck type equation which on one side maintains most of the properties of the solution to the kinetic collisional model, and on the other side is suitable to obtain the analytic expression of the steady solution in terms of the underlying parameters. This method is particularly suitable for trades which contain random parameters. Let us consider trade coefficients $p_{i}$ and $q_{i}$ in (2.11) defined as in the CPT model [11], namely

$$
p_{1}=1-\gamma+\eta, \quad q_{1}=\gamma, \quad p_{2}=\gamma, \quad q_{2}=1-\gamma+\eta_{*},
$$

where $\gamma<1 / 2$ measures, via its complement to unity, the common saving propensity of the transacting agents, and $\eta, \eta_{*}$ are random variables, representing, according to an idea of [11], the market returns of an open economy, and both taking values in the open interval $(-1+\gamma, 1-\gamma)$, with zero mean and equal variance $\sigma^{2}$, namely

$$
\langle\eta\rangle=\left\langle\eta_{*}\right\rangle=0, \quad\left\langle\eta^{2}\right\rangle=\left\langle\eta_{*}^{2}\right\rangle=\sigma^{2} .
$$

In this way the transaction operation, free from taxation and redistribution, is conservative in the mean, but not necessarily point-wise conservative (at the microscopic scale).

We may repeat now here the same procedure of [11] in order to construct the weak form of the kinetic equation governing the whole process and to derive the asymptotic limit of a continuous trading. Setting $\tau=\gamma t$ as new time variable, the weak form for the scaled unknown $g(v, \tau)$ turns out to be

$$
\begin{aligned}
& \frac{d}{d \tau} \int_{\mathbb{R}_{+}} g(v) \phi(v) d v \\
= & -\frac{\epsilon}{\gamma} \int_{\mathbb{R}_{+}}[\chi v-(\chi+1) m] g(v) \phi^{\prime}(v) d v \\
& +\int_{\mathbb{R}_{+}^{2}}\left[(w-v)-\frac{\epsilon}{\gamma} v\right] \phi^{\prime}(v) g(v) g(w) d v d w \\
& +\frac{1}{2} \int_{\mathbb{R}_{+}^{2}}\left[\gamma(w-v)^{2}+\frac{\sigma^{2}}{\gamma} v^{2}+\frac{\epsilon^{2}}{\gamma} v^{2}-2 \epsilon v(w-v)\right] \phi^{\prime \prime}(v) g(v) g(w) d v d w .
\end{aligned}
$$


Consider the following hydrodynamic limit:

$$
\sigma^{2} \rightarrow 0, \quad \gamma \rightarrow 0, \quad \epsilon \rightarrow 0, \quad \frac{\sigma^{2}}{\gamma} \rightarrow \lambda, \quad \frac{\epsilon}{\gamma} \rightarrow \kappa .
$$

In this regime, repeating the same steps as in [11] we get for $g(v, \tau)$ the following Fokker-Planck equation:

$$
\frac{\partial g}{\partial \tau}=\frac{\partial}{\partial v}\left\{\frac{\lambda}{2} v \frac{\partial}{\partial v}(v g)+\left[\left(\frac{\lambda}{2}+\kappa(\chi+1)+1\right) v-(\kappa(\chi+1)+1) m\right] g\right\},
$$

where the taxation/redistribution mechanism is represented solely by the positive parameter $\kappa$, which represents the limiting ratio of taxation rate and transaction coefficient, and by the constant $\chi \geq-1$ describing the redistribution policy. Clearly such an equation is an approximation of the Boltzmann kinetic equation, but, in the regime defined by (5.4), it describes the asymptotic limit which we are interested in. The steady state for the equation (5.5) is

$$
g_{\infty}(v)=\frac{(r m)^{1+r}}{\Gamma(1+r)} v^{-[2+r]} \exp \left\{-\frac{r m}{v}\right\}
$$

where $\Gamma$ denotes Euler gamma function [1], and

$$
r=\frac{2[\kappa(\chi+1)+1]}{\lambda} .
$$

We have $g_{\infty}(0)=0$, while for $v \rightarrow \infty$ we get $g_{\infty}(v)=O\left(v^{-(2+r)}\right)$. Therefore the Pareto index is here $1+r$, or, in other words, the moment of order $n$ exists finite if $n<1+r$. The CPT model of [11] is recovered for $\kappa=0$. The effects of taxation and redistribution are accounted for and explicitly quantified by the additional exponent $-2 \kappa(\chi+1) / \lambda$ for the wealth variable, which always makes tails slimmer, and thus wealth distribution more fair. It is clear that this effect is the stronger the higher is the factor $\kappa$, i.e. the higher the taxation rate. It also evident that, if $\kappa$ and $\lambda$ are fixed, tails become less and less thick as $\chi$ increases, and in all cases with $\chi>-1$, in which poorest people are favored by the new mechanism, the percentage of agents at high wealth decreases, whether or not they are in turn favored (as it would occur when $\chi>0$ ). No effects on the wealth distribution curve are present for $\chi=-1$, in which case the same index as for the CPT model [11] is recovered, independently of $\kappa$, namely, no matter how high is taxation. It is worth to mention, as a final remark, that $g_{\infty}(v)$ has a maximum at $\bar{v}=\frac{r m}{r+2}<m$ (most likely wealth smaller than average wealth). The height of the maximum is $\frac{1}{m} F(r)$, where

$$
F(r)=\frac{\exp \{(r+2)[\log (r+2)-1]\}}{r \Gamma(r+1)}
$$

diverges for $r \rightarrow 0^{+}$and $r \rightarrow+\infty$, with a positive minimum at $r=\bar{r}$, where $\bar{r}$ is the solution of the transcendental equation

$$
\Psi(r+1)=\log (r+2)-\frac{1}{r},
$$

with $\Psi$ denoting the digamma function [1]. The result is $\bar{r} \simeq 2.4825$, indicating a universal optimal value for flattening wealth distribution that can be achieved by playing properly with the taxation parameter $\epsilon$, once the others are known. 


\section{Continuous trading limit of a model without risk}

Another significant continuous trading limit is in order when no risks (no random variables) are present in the model, which simply amounts to setting $\sigma=0$ in the rescaled weak form (5.3). The case of no taxation/redistribution, considered by Pareschi and Toscani in [22], corresponds to the further option $\epsilon=0$. It is clear that in the latter situation binary transactions are pointwise conservative, and therefore it is well known [22] that wealth distribution approaches a steady state in which all agents share the same (constant) average wealth $m$ (no tails at all in this frame). Let us add taxation and redistribution in an overall conservative fashion, as before, and let us consider the limiting situation in which not only $\gamma$ and $\epsilon$ are vanishingly small, but also $\chi$ is vanishingly greater than -1 , and the mutual relationships are defined by the hydrodynamic limit

$$
\gamma \rightarrow 0, \quad \epsilon \rightarrow 0, \quad \frac{\epsilon^{2}}{\gamma} \rightarrow \lambda, \quad \frac{\chi+1}{\epsilon} \rightarrow \beta>0 .
$$

In this regime, we get for $g(v, \tau)$ the Fokker-Planck equation:

$$
\frac{\partial g}{\partial \tau}=\frac{\partial}{\partial v}\left\{\frac{\lambda}{2} v \frac{\partial}{\partial v}(v g)+\left[\left(\frac{\lambda}{2}+\lambda \beta+1\right) v-(\lambda \beta+1) m\right] g\right\},
$$

similar to but different from (5.5), whose solution is given again by (5.6), where now the parameter $r$ is given by

$$
r=\frac{2(\lambda \beta+1)}{\lambda}
$$

The role of the parameter $\lambda$ is played here by the limiting ratio $\varepsilon^{2} / \gamma$ (so, $\gamma$ must be $O\left(\varepsilon^{2}\right)$ for $\varepsilon \rightarrow 0$ ) instead of the limiting ratio $\sigma^{2} / \gamma$ which is in order for (5.5), and taxation/redistribution affects the final result also through the additional limiting factor $\beta$ (implying $\chi=-1+O(\varepsilon)$ ). The same considerations as before are in order, mutatis mutandis. In particular, the steady state solution exhibits a Pareto tail $O\left(v^{-(2+r)}\right)$, and the additional power that makes tail slimmer with respect to the CPT model [11] is exactly $-2 \beta$, which is independent here from $\lambda$. The most remarkable fact is however that the taxation/redistribution mechanism causes a radical change in the shape of the equilibrium wealth distribution, which is transformed from a sharp delta measure to a smooth positive function with support on all of $\mathbb{R}_{+}$, and with fat Pareto tail for $v \rightarrow \infty$. On the other hand this is in agreement with the kind of redistribution relevant to the considered parameters $(\chi \simeq-1)$, which enhances the richest and the poorest parts of the agent population, and penalizes the intermediate part around the mean wealth.

Another approach to the same problem might resort to the Laplace transform of the kinetic equation, according to the line of [25] presented in section 4 (see also [14]). For general parameters $\gamma, \epsilon$, and $\chi$ the equation for the transform $\tilde{f}(s, t)$ reads as

$$
\frac{\partial \tilde{f}}{\partial t}+\epsilon\left[\chi s \frac{\partial \tilde{f}}{\partial s}+(\chi+1) m s \tilde{f}\right]=\tilde{f}((1-\gamma-\epsilon) s) \tilde{f}(\gamma s)-\tilde{f}(s) .
$$

It is easily seen that, in the continuous trading limit defined by (6.1), the stationary version of this nonlocal equation takes the local form of a second order ordinary differential equation

$$
\frac{\lambda}{2} s \frac{d^{2} \tilde{f}}{d s^{2}}=(\lambda \beta+1)\left[\frac{d \tilde{f}}{d s}+m \tilde{f}(s)\right]
$$


which is amenable, in terms of the new variable $z=2(\mathrm{mrs})^{1 / 2}$, to a modified Bessel equation of order $1+r$. Skipping known details, its physical solution, involving the Bessel function $K_{1+r}(z)$, may be Laplace inverted and leads back, for the steady wealth distribution, to the same solution (5.6) of the Fokker-Planck equation (6.2).

In the present conservative regime without risks, information on the stationary equilibrium distribution may be achieved for general values of parameters also from the evolution of moments, which is governed by the weak form of the kinetic equation relevant to the test function $\phi(v)=v^{n}$, that is readily seen to read as

$$
\begin{aligned}
& \frac{d}{d t} \int_{\mathbb{R}_{+}} v^{n} f(v) d v-\epsilon \int_{\mathbb{R}_{+}} v^{n} \frac{\partial}{\partial v}\{[\chi v-(\chi+1) m] f\} d v \\
= & \int_{\mathbb{R}_{+}^{2}}\left[\left(v^{*}\right)^{n}-v^{n}\right] f(v) f(w) d v d w .
\end{aligned}
$$

Since $v^{*}=(1-\gamma-\epsilon) v+\gamma w$, the integral on the right hand side may be recast as

$$
\begin{aligned}
& \int_{\mathbb{R}_{+}^{2}}\left[\left(v^{*}\right)^{n}-v^{n}\right] f(v) f(w) d v d w \\
= & \sum_{k=1}^{n-1}\left(\begin{array}{l}
n \\
k
\end{array}\right)(1-\gamma-\epsilon)^{n-k} \gamma^{k} M_{n-k} M_{k}+\left[(1-\gamma-\epsilon)^{n}+\gamma^{n}-1\right] M_{n},
\end{aligned}
$$

where

$$
M_{j}=\int_{\mathbb{R}_{+}} v^{j} f(v) d v,
$$

while the other collision contribution due to $R_{\chi}^{\epsilon}$ is simply handled by parts to finally yield

$$
\begin{gathered}
\frac{d M_{n}}{d t}+\epsilon n\left[\chi M_{n}-(\chi+1) m M_{n-1}\right] \\
=\sum_{k=1}^{n-1}\left(\begin{array}{l}
n \\
k
\end{array}\right)(1-\gamma-\epsilon)^{n-k} \gamma^{k} M_{n-k} M_{k}+\left[(1-\gamma-\epsilon)^{n}+\gamma^{n}-1\right] M_{n} .
\end{gathered}
$$

Therefore, moments of the steady state are related by the recursive formula

$$
\begin{gathered}
{\left[1-(1-\gamma-\epsilon)^{n}-\gamma^{n}+\epsilon \chi n\right] M_{n}^{\infty}} \\
=\epsilon(\chi+1) m n M_{n-1}^{\infty}+\sum_{k=1}^{n-1}\left(\begin{array}{l}
n \\
k
\end{array}\right)(1-\gamma-\epsilon)^{n-k} \gamma^{k} M_{n-k}^{\infty} M_{k}^{\infty} .
\end{gathered}
$$

As long as the coefficient of $M_{n}^{\infty}$ on the left hand side is different from zero, this formula allows us to compute a moment of any order $n$ in terms of all lower order moments, starting from $M_{0}^{\infty}=1$ and $M_{1}^{\infty}=m$, even in absence of an explicit knowledge of the steady distribution. If $\chi \geq 0$, all coefficients of $M_{n}^{\infty}$ are positive, for any $n>0$, which indicates that all moments of $g_{\infty}$ are well defined and the steady wealth distribution has slim tails. On the other hand, if $-1 \leq \chi<0$, the redistribution regime to which the previously considered asymptotic limit (6.1) applies, the coefficient of $M_{n}^{\infty}$ becomes negative sooner or later for increasing $n$, with a breakdown in the procedure (all moments are bound to be positive), meaning lack of higher order moments, and thus implying fat Pareto-like tail. Again, this is not surprising, since redistribution favors in part also the richest fraction of agents when $\chi<0$, whereas it increasingly penalizes such fraction for increasing positive $\chi$. 


\section{Conclusions}

In this paper, we introduced and discussed kinetic models for wealth distribution in a simple market economy, which are able to reproduce the salient features of the wealth distribution by including taxes to each trading process and redistributing the collected money among the population according to a given criterion. This model of redistribution allows movement of the money obtained from taxation of trades in a uniform way among agents of the market, as well as concentration of the wealth around the (constant) mean wealth. Each of these operations can be quantified by a rate parameter which is responsible of the steady profile. Our analysis gives a theoretical basis to some recent research $[24,19,16]$, that analyzed discrete simplified models for the exploitation of finite resources by interacting agents, where each agent receives a random fraction of the available resources. Both numerical experiments and asymptotic analysis show that in general the redistribution is able to modify the Pareto index, and that this modification can be quantified in terms of the redistribution operator. Future scheduled work includes numerical implementation of the present model, taxation mechanisms which depend on the wealth of the agent or on the amount of money earned by transaction, and possibly comparison of our results with empirical data.

Acknowledgement. This work has been sponsored by Fondazione Cariparma (Parma, Italy), Grant No. 2008/0256, project "Kinetic models for socio-economic phenomena", that provided financial coverage for the research contract of one of the authors (MB). All authors also acknowledge support from the Italian MIUR, project "Kinetic and hydrodynamic equations of complex collisional systems", and from GNFM-INdAM, project "Fokker-Planck equations with superlinear drift".

\section{REFERENCES}

[1] M. Abramowitz and I.A. Stegun, Handbook of Mathematical Functions, Dover, New York, 1972.

[2] R.A. Adams and J.J.F. Fournier, Sobolev Spaces, Academic Press, Amsterdam, 2003.

[3] A.V. Bobylev, The theory of the spatially uniform Boltzmann equation for Maxwell molecules, Sov. Sci. Review C, 7, 112-229, 1988.

[4] J.P. Bouchaud and M. Mézard, Wealth condensation in a simple model of economy, Physica A, 282, 536-545, 2000.

[5] A. Chakraborti, Distributions of money in models of market economy, Int. J. Modern Phys. C, 13, 1315-1321, 2002.

[6] A. Chakraborti and B.K. Chakrabarti, Statistical mechanics of money: effects of saving propensity, Eur. Phys. J. B, 17, 167-170, 2000.

[7] A. Chatterjee, B.K. Chakrabarti and S.S. Manna, Pareto Law in a kinetic model of market with random saving propensity, Physica A, 335, 155-163, 2004.

[8] A. Chatterjee, S. Yarlagadda, B.K. Chakrabarti (Eds.) Econophysics of Wealth Distributions, New Economic Window Series, Springer-Verlag, (Italy), 2005.

[9] A. Chatterjee, B.K. Chakrabarti and R.B. Stinchcombe, Master equation for a kinetic model of trading market and its analytic solution, Phys. Rev. E, 72, 026126, 2005.

[10] S. Cordier, L. Pareschi and C. Piatecki, Mesoscopic modelling of financial markets, J. Stat. Phys., 134, 161-184, 2009.

[11] S. Cordier, L. Pareschi and G. Toscani, On a kinetic model for a simple market economy, J. Stat. Phys., 120, 253-277, 2005.

[12] A. Drăgulescu and V.M. Yakovenko, Statistical mechanics of money, Eur. Phys. Jour. B, 17, 723-729, 2000.

[13] B. Düring, D. Matthes and G. Toscani, Kinetic equations modelling wealth redistribution: a comparison of approaches, Phys. Rev. E, 78, 056103, 2008.

[14] B. Düring, D. Matthes and G. Toscani, A Boltzmann type approach to the formation of wealth distribution curves, Riv. Mat. Univ. Parma, Series 8, Vol. 1, 199-261, 2009. 
[15] U. Garibaldi, E. Scalas and P. Viarengo, Statistical equilibrium in simple exchange games II. The redistribution game, Eur. Phys. Jour. B, 60, 241-246, 2007.

[16] S. Guala, Taxes in a simple wealth distribution model by inelastically scattering particles, arXiv: 0807.4484v1, preprint, 2008.

[17] B. Hayes, Follow the money, American scientist, 90, 400-405, 2002.

[18] S. Ispolatov, P.L. Krapivsky and S. Redner, Wealth distributions in asset exchange models, Eur. Phys. Jour. B, 2, 267-276, 1998.

[19] J.R. Iglesias, S. Gonçalves, S. Pianegonda, J.L. Vega and G. Abramson, Wealth redistribution in our small world, Physica A, 327, 2003.

[20] O. Malcai, O. Biham, P. Richmond and S. Solomon, Theoretical analysis and simulations of the generalized Lotka-Volterra model, Phys. Rev. E, 66, 031102, 2002.

[21] D. Matthes and G. Toscani, On steady distributions of kinetic models of conservative economies, J. Stat. Phys., 130, 1087-1117, 2008.

[22] L. Pareschi and G. Toscani, Self-similarity and power-like tails in nonconservative kinetic models, J. Stat. Phys., 124, 747-779, 2006.

[23] V. Pareto, Cours d'Economie Politique, Lausanne and Paris, 1897.

[24] S. Pianegonda, J.R. Iglesias, G. Abramson and J.L. Vega, Wealth redistribution with finite resources, Physica A, 322, 667-675, 2003.

[25] F. Slanina, Inelastically scattering particles and wealth distribution in an open economy, Phys. Rev. E, 69, 046102, 2004.

[26] S. Solomon and P. Richmond, Stable power laws in variable economies; Lotka-Volterra implies Pareto-Zipf, Eur. Phys. J. B, 27, 257-262, 2002. 